P71 (continued)

Correlation Coefficient. Statistical significance was set at $\mathrm{p}=0.05$.

Results: Accelerometer derived MPA $(\mathrm{r}=0.2, \mathrm{P}=0.02)$, VPA $(\mathrm{r}=0.3, \mathrm{P}=0.003)$, and MVPA $(\mathrm{r}=0.3, \mathrm{P}=0.008)$ were positively correlated with quality of life. There were no associations between ST or LPA and quality of life. Of the 4 subscales child physical functioning was positively correlated with MPA $(\mathrm{r}=0.3, \mathrm{P}=0.005)$, VPA $(\mathrm{r}=0.3$, $\mathrm{P}=0.0006)$, and MVP $(\mathrm{r}=0.3, \mathrm{P}=0.001)$; child school functioning with MPA, $(\mathrm{r}=0.2, \mathrm{P}=0.01)$, VPA $(\mathrm{r}=0.3, \mathrm{P}=0.005)$, and MVPA $(\mathrm{r}=0.3, \mathrm{P}=0.005)$. There were no associations between subscales emotional and social functioning and PA.

Conclusions and Implications: Inclusion of quality of life dimensions into obesity prevention programs that increase physical activity in 9-10 year olds warrants further exploration.

Funding: USDA

\section{P72 Trends in Fruits and Vegetables Intake Among Older Adults in North Carolina: Gender and Ethnic Differences}

Oyinlola Babatunde, PhD, RD, MPH, babatundeo@ecu.edu, East Carolina University, RW-331 Department of

Nutrition Science, Mail Stop \#505, Greenville, NC, 27858; S. Imai, PhD

Objective: The objective of this study was to determine the trend in daily fruits or vegetables (FV) intake among older adults in North Carolina and if this differs by gender, or race/ethnicity.

Study Design, Setting, Participants, and Intervention: Cross sectional study design, using 20022009 North Carolina Behavioral Risk Factors Surveillance System (BRFSS) data. BRFSS is part of a large national survey on health and health risk factors. Participants were a total of 38,081 older adults, $=60$ years.

Outcome, Measures and Analysis: Consumption of at least three servings FV daily, gender and ethnicity. Descriptive statistics and confidence intervals (CI) were used to establish trends.

Results: Percent of older adults $=60$ years consuming $=3$ servings of FV daily decreased from $68.8 \%(\mathrm{CI}=65.44-$ $71.93)$ in 2002 to $62 \%(\mathrm{CI}=59.97-63.97)$ in 2009. Males had lower intake overall compared to females (59 and $64 \%$ respectively). In 2009, non-Hispanic blacks had the lowest intakes (52.1\%), compared to 57.9\% in Hispanic, $63.1 \%$ in other race/ethnicity and $63.9 \%$ in non-Hispanic white.

Conclusions and Implications: FV consumption is essential for healthy aging, especially in the prevention, reduction of risks, and management of several chronic diseases. These findings support the need for culturally appropriate strategies that help improve FV intake in this vulnerable and at-risk population, particularly among racial/ethnic minorities within the group.

Funding: None

\section{P73 Head Start Administrator and Teacher Perceptions of Parental Influence on Preschool Children}

Oyinlola Babatunde, PhD, RD, MPH, babatundeo@ecu.edu, East Carolina University, RW-331 Department of Nutrition Science, Mail Stop \#505, Greenville, NC, 27858; K. N. Wilkerson; S. E. Lisson; V. Carraway-Stage, PhD, RD, LDN; O. T. Babatunde, PhD, RD, MPH

Objective: The purpose of this study was to explore parental influence on preschool children's nutrition education from the perspective of Head Start administrators and teachers.

Study Design, Setting, Participants, and Intervention: Beginning September 2011 through May 2012, researchers conducted 63 in-depth, structured interviews with North Carolina-based (NC) Head Start Health/Nutrition Coordinators $(\mathrm{n}=31)$ and teachers $(\mathrm{n}=32)$.

Outcome, Measures and Analysis: Researchers recorded interviews via digital audio format and transcribed them verbatim. Through open-coding, researchers identified four primary, emergent themes related to parental influence. Following a grounded theory approach, themes were then broken down to develop a substantive-level model.

Results: Outcomes revealed parent interaction involvement (e.g. parent meetings), educational opportunities offered through Head Start (e.g. parent education classes), community support programs (e.g. public funding, private organizations), and factors related to a parent's own background (e.g. socio-economic status, food preferences) were principal parent related-components that Head Start staff reported as being influential to preschooler's nutrition education.

Conclusions and Implications: Initial findings suggest more training and education opportunities for parents may be needed to overcome their own nutrition-related knowledge and perceptions. Yet, participants often reported a lack of parental involvement as a barrier to educational opportunities offered to families through Head Start. Despite barriers, teachers and local community partners should continue supporting parents in efforts to reinforce positive nutrition messages among preschool children. However, in order to better understand the impact of parent-related factors (e.g. involvement, influence) on children's nutrition education, researchers should also explore these issues from the perspective of Head Start parents.

Funding: None 